\title{
BMJ Open Lending an Ear: iPeer2Peer plus Teens Taking Charge online self-management to empower adolescents with arthritis in Ireland: protocol for a pilot randomised controlled trial
}

\author{
Siobhan O'Higgins, ${ }^{1}$ Jennifer Stinson, ${ }^{2,3}$ Sara Ahola Kohut, ${ }^{2,4}$ Line Caes, ${ }^{5}$ \\ Caroline Heary, ${ }^{6}$ Brian E McGuire, ${ }^{1}$ Team Pain, Centre for Pain Research, School of \\ Psychology, NUI Galway
}

To cite: 0 'Higgins S, Stinson J, Ahola Kohut S, et al. Lending an Ear: iPeer2Peer plus Teens Taking Charge online self-management to empower adolescents with arthritis in Ireland: protocol for a pilot randomised controlled trial. BMJ Open 2019;9:e27952. doi:10.1136/ bmjopen-2018-027952

- Prepublication history for this paper is available online. To view these files please visit the journal online (http://dx.doi org/10.1136/bmjopen-2018027952).

Received 16 November 2018 Revised 12 July 2019 Accepted 18 July 2019
Check for updates

(C) Author(s) (or their employer(s)) 2019. Re-use permitted under CC BY-NC. No commercial re-use. See rights and permissions. Published by BMJ.

For numbered affiliations see end of article.

Correspondence to Dr Siobhan O'Higgins; siobhan.ohiggins@nuigalway.ie

\section{ABSTRACT}

Introduction Juvenile idiopathic arthritis (JIA) negatively affects adolescents' everyday activities. To address the need for innovative, effective, convenient, lowcost psychosocial self-management programmes, we developed an Irish version of Canadian Teens Taking Charge (TTC) and integrated it with Skype-based peer support iPeer2Peer (iP2P).

Objectives To explore the feasibility and preliminary outcome impact (effectiveness) of an integrated iP2P and Irish TTC, via three-arm (treatment as usual, TTC and $\mathrm{P} 2 \mathrm{P}-\mathrm{TTC}$ ) pilot randomised controlled trial (RCT); and determine feasibility and sample size for a full RCT. To ensure active involvement of adolescents with JIA via a Young Person Advisory Panel and examine how participants experienced the study. Finally, to see if TTC and IP2P with TTC reduce costs for families.

Methods and analysis Recruitment of 60 families will be ongoing until July 2019, via healthcare professionals and support groups. Analysis will consist of single-blinded (outcome assessment), three-arm pilot RCT, using online questionnaires, with assessments at baseline (T1), after intervention (T2) and 3 months post-intervention (T3). The primary outcomes on feasibility with comparisons of TTC and P2P-TTC on fidelity, acceptability and satisfaction, engagement and degrees of tailoring. The secondary outcomes will be self-management and self-efficacy and a range of health-related quality-of-life factors, pain indicators and costs.

Participants from the intervention groups will be invited to share their perspectives on the process in semistructured interviews. Quantitative data will be analysed using SPSS V.21 and the audio-taped and transcribed qualitative data will be analysed using qualitative content analysis.

Dissemination Via journal articles, conference presentations, co-delivered by key stakeholders when possible, launch of accessible, effective and sustainable Internet self-management and peer support for Irish adolescents with JIA.

Trial registration number ISRCTN13535901; Pre-results.
Strengths and limitations of this study

- This study follows from an in-depth qualitative exploration of the need and desire among stakeholders for an online support programme.

- The needs analysis offered suggestions on how to adapt the Canadian programme to ensure that the programme offered relevant information to the Irish context; these changes have been achieved.

- Canadian Teens Taking Charge (TTC) is known to improve the lives of teens with juvenile idiopathic arthritis and their families; this study builds on that success by adding extra elements of tailoring and sustainability with the iPeer2Peer mentoring.

- Sustainability of the integrated programme, if found to be effective, is ensured due to ongoing collaboration with all stakeholders; Arthritis Ireland (a nonprofit support service for people with arthritis) taking over the peer mentoring element of the intervention, with ongoing training and support for mentors; and TTC will be updated biannually by About Kids Health in Toronto and healthcare professionals in Ireland. Recruitment of sufficient teens and their families for the randomised controlled trial may be difficult, hence active involvement of all stakeholders and the Young Person Advisory Panel will be crucial to reach our target sample size.

\section{INTRODUCTION}

Juvenile idiopathic arthritis (JIA) is the most common childhood rheumatic disease. In Ireland, 1200 children live with JIA, with over 100 children newly diagnosed annually. ${ }^{1}$ Children and adolescents commonly experience a myriad of physical and emotional symptoms that restrict physical and social interactions and negatively impact their health-related quality of life (HRQL). ${ }^{23}$ There is no cure, the disease course can be unpredictable, and HRQL deteriorates with increased disease 
severity, active joint counts, pain and degree of disability. ${ }^{23}$ The Irish rheumatologist:patient ratio for children with arthritis is second lowest in Europe, with waiting lists of up to 2 years; access to psychological support is equally limited. In addition, transition to adult services is scheduled by age 16 years, rather than 18 as in Canada. So, although cognitive-behavioural therapy interventions can lead to improvement in pain and HRQL, ${ }^{4}$ most teens with JIA in Ireland will not receive these interventions although eventually they may avail of them through the adult rheumatology services, hence, the need for supports to develop self-management skills for adolescents with JIA. Using the internet is a possible solution to address the gap between need, availability and access to effective treatments. Online interventions are scalable and accessible in the moment, 24 hours a day, and do not need therapist involvement.

Prior to this study, our Canadian colleagues evaluated the two programmes in the present study separately. Stinson and colleagues developed and tested the usability, feasibility and effectiveness of Teens Taking Charge (TTC), an online self-management programme consisting of 12 modules for teens and 2 for parents, with telephone support from a health coach (trained, adult non-healthcare professionals (HCPs) without arthritis) for Canadian adolescents with JIA. ${ }^{3} 56$ Significant improvements were found in disease-related knowledge, decreased pain and increased exercise adherence. ${ }^{6}$ Peer support by another person with similar chronic illness is associated with improved health outcomes. ${ }^{7-10}$ iPeer2Peer (iP2P), an online peer mentoring programme, was evaluated with adolescents with chronic pain, and found to improve acceptability of self-management and peer support treatments. ${ }^{11}$ Although positively evaluated separately, the two programmes have not been combined before.

Localising available and effective programmes which meet the specific needs of patients is an important development strategy for facilitating timely availability of evidence-based programmes. ${ }^{12}$ Hence, we conducted an Irish qualitative needs assessment working with members (teens and parents) of two patient organisations: Arthritis Ireland (AI) and Irish Children's Arthritis Network (iCAN, a support group set up by a parent for other parents and teens with JIA); HCPs from Our Lady's Children's Hospital (OLCH) Crumlin and other paediatric units. The interviews explored stakeholder perspectives on

- Impact of JIA on adolescents and families.

- Current Irish service provision.

- The value and usability of Canadian 'TTC: Managing Arthritis Online' and iP2P programmes.

Lack of access to local multidisciplinary rheumatology teams was the main concern of all stakeholders. There was consensus that TTC would be a useful resource once TTC information was tailored to the Irish context and specific needs of each patient, and facilitated through peer mentoring. ${ }^{13}$
These views, taken together, underpinned the critical need for accessible and effective interventions to assist Irish adolescents with JIA to find effective ways to selfmanage symptoms and improve overall HRQL. iP2P mentoring combined with TTC also has the potential to reduce the burden on services, by providing information, support and empowerment tailored to the teens' in- themoment needs, reducing the need to contact a HCP.

Based on our qualitative need assessment, and through further consultations with HCPs, support services AI and iCAN, the five teens with JIA who make up our Young Person Advisory Panel (YPAP), and their parents, the Canadian TTC website's three components which are encapsulated within the 12 modules, have been culturally adapted:

1. Disease-specific content (what is JIA, how is it diagnosed, how is it treated using pharmacological, physical and psychological strategies);

2. Developing self-management skills to live well with JIA (managing emotions, managing physical symptoms, healthy lifestyle, skills to move on to adult healthcare, education and vocational skills to manage JIA);

3. Social support (videos and stories of hope).

The Irish TTC has videos of Irish teens with JIA and their parents and HCP talking about the different topics, integrated with videos of Canadians, throughout the 12 modules. The text has been adapted with relevant drugs, service and financial support offered in Ireland rather than those in Canada. This process has taken a year to achieve and Sick Kids Hospital adapted the online TTC to incorporate the changes that ensure the Irish TTC is acceptable and relevant to Irish families. The development of the pilot RCT can be seen in figure 1 .

This study will examine if greater reach and adoption of self-management and peer support programmes are achievable using novel information and communication technologies (ie, e-Health), shown in figures 2 and $3 .{ }^{14}$

\section{Objectives}

- Explore feasibility and preliminary outcome impact (effectiveness) of an integrated iP2P and Irish TTC, via three-arm (treatment as usual, TTC and iP2PTTC) pilot RCT of 'Teens Taking Charge: Managing Arthritis Online' to help adolescents with JIA improve their self-management skills, HRQL, disease knowledge, social support, self-efficacy, physical symptoms and emotional distress, compared with a treatment-asusual control group.

- Determine feasibility and sample size for a full RCT.

- Ensure active involvement of adolescents with JIA throughout the study with creation and support of a YPAP, who communicate monthly with the research team via Zoom calls and WhatsApp group messaging. They and their parents reviewed the videos and text of the Irish TTC, posters and all recruitment information sent out to parents and teens.

- To examine how participants experienced the study 


\section{Steps in Development, Evaluation and Future Plans}

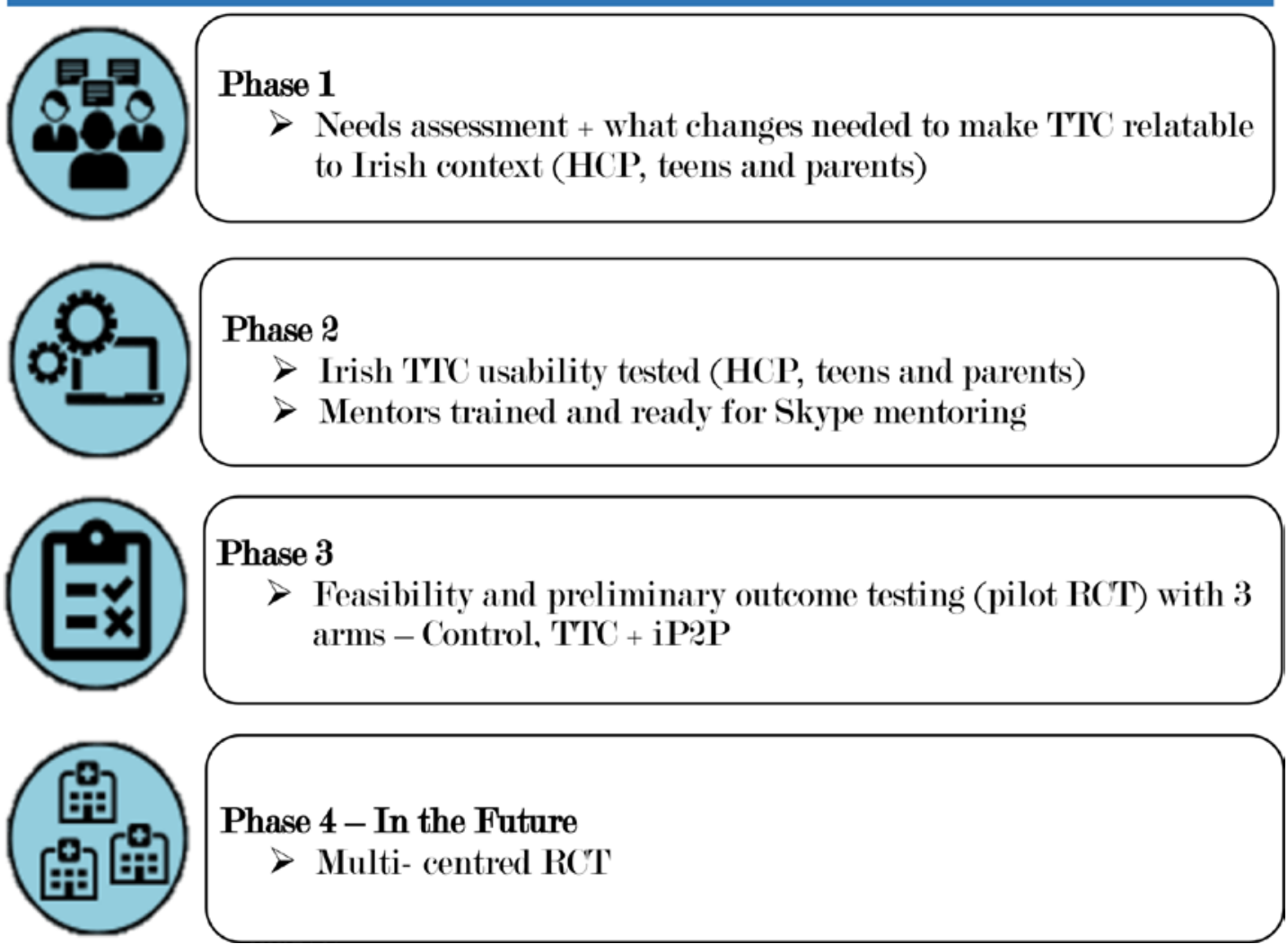

Figure 1 Development of pilot randomised controlled trial (RCT) and future for full RCT of Irish Teens Taking Charge (TTC) and iPeer2Peer (iP2P). HCP, healthcare professional.

- To see if TTC and iP2P and TTC reduce costs for families of teens with JIA.

\section{Hypotheses}

1. A feasible Internet self-management programme alongside a peer support intervention will provide Irish adolescents with JIA evidenced-based arthritis self-management and transitional care knowledge and skills; as well as the possibly of reducing $\mathrm{HC}$ costs for families.

2. The involvement of the YPAP, AI and iCAN will support a successful, sustainable and adolescent-appropriate launch of the adjusted Irish TTC and peer support programme.

\section{METHODS AND ANALYSIS}

\section{Patient and public involvement}

Before designing the pilot RCT, we worked with adolescents with JIA, their parents and HCP to explore their experiences of living with JIA, levels of support and medications within the Irish system and explored whether interventions such as TTC and iP2P would be welcomed and useful to them. ${ }^{13}$

Once we knew that the interventions would be positively received and of use to our target population, we invited adolescents and their parents to be part of a
Young Persons' Advisory Panel (YPAP) from the start of the research process.

All stakeholders were involved in the adaption of the online programme, recruitment of the mentors and trialling of the adapted online programme, design of recruitment posters and the measures for the online before and after the intervention. Hosted by LimeSurvey.

The YPAP met initially-courtesy of their parents bringing them to a central location-for a day's training. The group meet every month either via WhatsApp or scheduled Zoom meetings; another face-to-face day is planned during school holidays.

See our dissemination plans for how study participants will be kept informed of the progress of the study and our results.

From the start of this process, stakeholders have reviewed several times the online intervention, and given feedback on how to ensure it is both acceptable and accessible to the Irish target population of teens with JIA and their parents.

\section{Study design}

A single-blinded (outcome assessment), pilot RCT design with three arms (20 teens in each) to test the feasibility and effectiveness of the Irish-adapted TTC with and without integrated iP2P intervention for a 12-week period. 

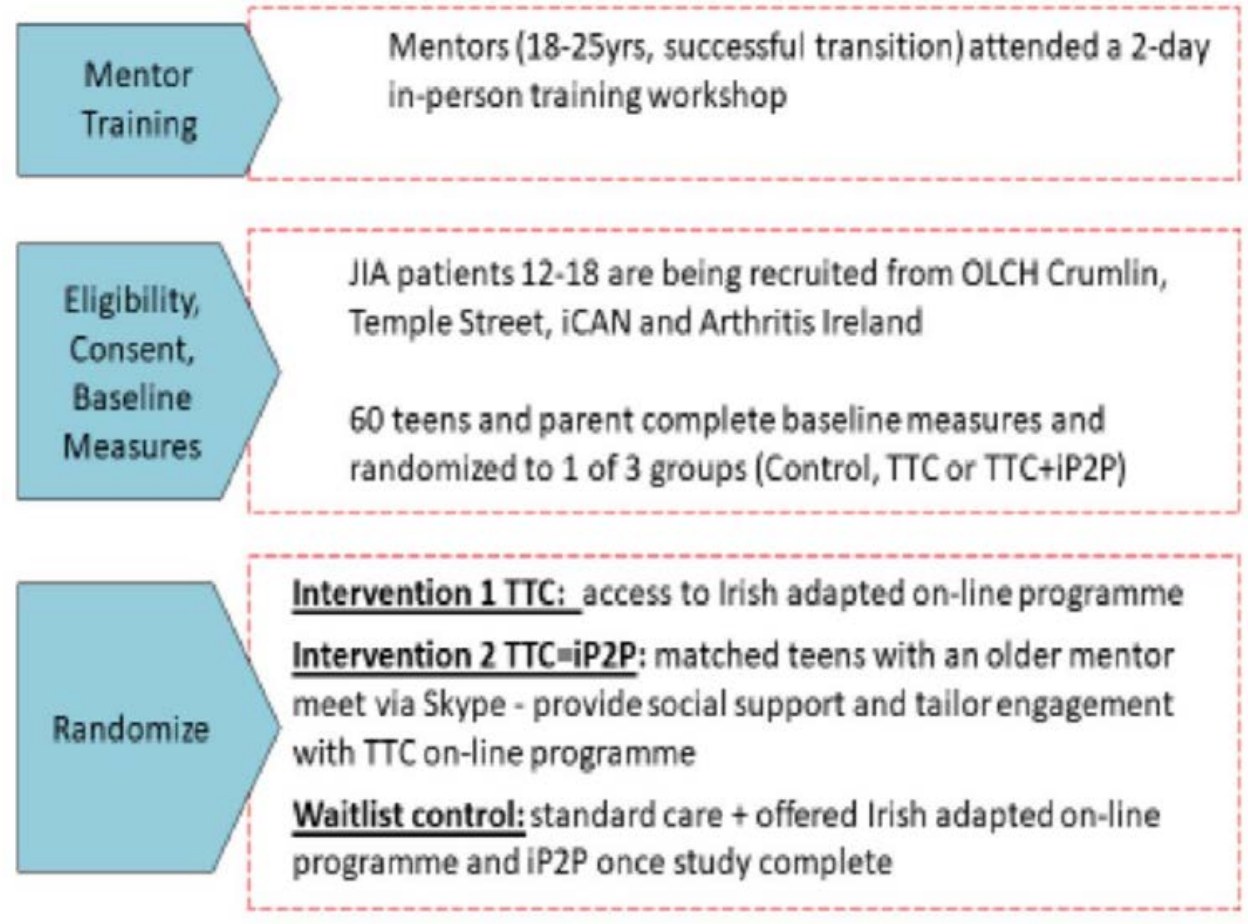

Endpoint

All participants (teens, parents and mentors ) complete endpoint

Measures measures and satisfaction feedback interview or focus group

Figure 2 Schema of pilot randomised controlled trial of Teens Taking Charge (TTC) with iPeer2Peer (iP2P). iCAN, Irish Children's Arthritis Network; JIA, juvenile idiopathic arthritis; OLCH, Our Lady's Children's Hospital.

\section{Participant eligibility}

Inclusion criteria

- Adolescents between 12 and 18 years old.

- Parental consent for teen to participate.

- Adolescents diagnosed with and actively being treated for JIA.

- Parent and adolescent both able to speak and read English.

- Access to a computer, smartphone or tablet capable of using free Skype software.

- Willing and able to complete online measures.

- Adolescent eligible to participate without participation of a parent.

Exclusion criteria

- Major cognitive impairments-based on medical assessments.

- Comorbid medical or psychiatric illnesses which may impact on ability to understand and use web-based programmes-based on medical assessments.

- Parents/caregivers not eligible to participate in the study without an adolescent.

\section{Recruitment}

Three recruitment avenues:

1. All registered and eligible patients of OLCH Crumlin Paediatric Rheumatology programme will be sent an invitation to participate by the HCP. Plus the research team will invite patients attending regularly scheduled clinic visits.

2. Both AI and iCAN will be asked to inform their members of the study.

3 . The study will be advertised through various social media channels (Facebook, Twitter etc).

For all routes of recruitment: once a parent emails or calls the Centre for Pain Research (CPR) for more information, this will be sent out by the research assistant (RA), with consent and assent forms for both parents and adolescent.

It is not anticipated that the three processes will recruit sufficiently different types of teens-although those recruited by the HCP may be those with a more recent diagnosis. Date of diagnosis will be noted in their demographic survey and on consent and assent forms for future reference.

All potential participants will be contacted, consent and assent forms as well as detailed information emailed or sent to each family, to be returned signed prior to randomisation.

Recruitment will continue until end of July 2019.

\section{Randomisation}

When a participant agrees to take part in the trial, they will all complete online T1 measures and then be randomly 


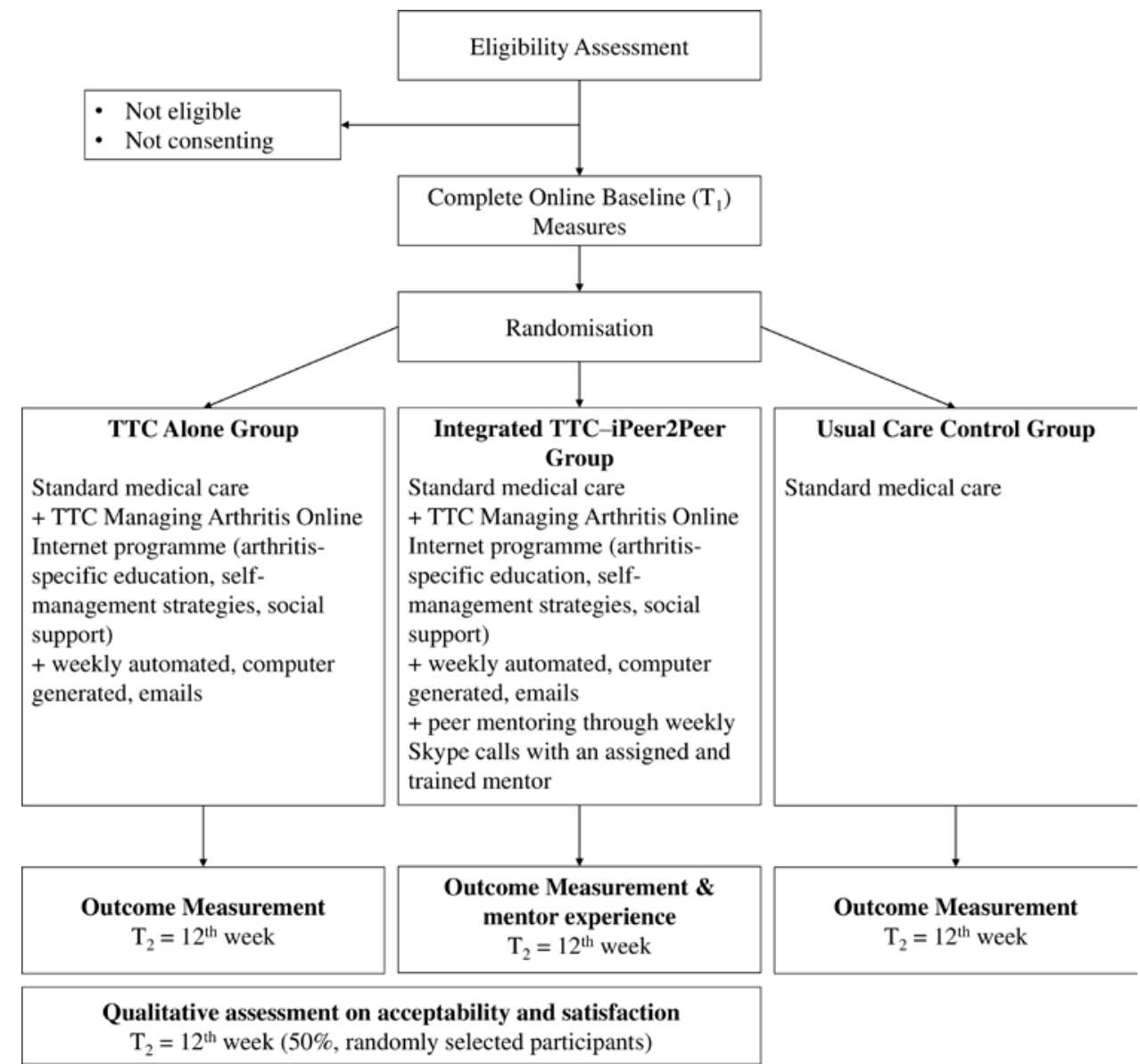

Figure 3 Flowchart of pilot randomised controlled trial. TTC, Teens Taking Charge.

assigned to one of the three arms using random permuted blocks to ensure balanced groups. Randomisation will be via a custom-written script, administered from a passwordsecured server, by the CPR's medical statistician. As such, researchers will not hold influence in the allocation process. Once assigned to a group, the specific details for involvement over the next 12 weeks will be emailed to them.

\section{Description of study arms}

1. Control group will receive usual healthcare appointments, medication and therapies, which may include physiotherapy, occupational therapy and talking with a psychologist (these are often organised by families rather than through the hospital).

2. Experimental groups.

For both intervention groups (TTC alone and iP2P-TTC)

In addition to standard medical care, the two experimental groups will receive 12 modules of interactive multicomponent self-guided online TTC intervention. There are also two modules specifically for parents/caregivers to help encourage healthy behaviour.

The TTC programme will be delivered on a restricted password-protected website allowing the team to track usage. TTC programme is set up in a modular fashion that participants work through over a 15-week period at their own pace. Adolescents will be encouraged to log onto the website and complete one module per week. However, website activity will be flexible, and adolescents will be able to catch up missed modules (eg, due to feeling unwell, examinations, holidays etc). A 91\% (20/22) compliance was achieved in the pilot RCT of the original TTC. ${ }^{6}$ Consequently, for both TTC alone and integrated iP2P-TTC groups, data from participants completing at least $70 \%$ of the TTC programme will be considered valid for analyses.

\section{iP2P-TTC}

As well as access to TTC, each participant will be matched with a peer mentor. Mentors identified by the HCP or the support groups (AI and iCAN) will have undergone 2 days of training and Gardaí vetting. They will have Skype calls with their mentees for up to an hour every week. There will be flexibility in number of sessions a dyad will have (in Canadian pilot RCT, males preferred fewer sessions). ${ }^{11}$ However, we will advise weekly contact. The aim will be for a maximum of 12 calls within 15 weeks.

\section{SAMPLE SIZE AND POWER ESTIMATIONS Pilot RCT}

It is suggested that between 20 and 30 participants be recruited per group for pilot studies to examine overall feasibility and for development of estimates (eg, variance) 
to compute power for a larger trial. ${ }^{1516}$ Therefore, we will recruit a total of 60 participants 12-18years old (20 in each arm) and their primary caregivers.

\section{Mentors for iP2P}

At least five participants 17-26years old will complete a previously validated 2-day training course (organised in collaboration between co-applicants, Drs Stinson and Kohut and collaborators AI and iCAN) and supported throughout the duration of the study (eg, consultations with research staff, additional training in mentorship skills if needed).

\section{Young Person Advisory Panel}

Five participants 12-20 years old following a day's training in research methods and exploration of their role as a team of experts, and meet, both face-to-face and via Zoom, regularly over the course of the study. Our commitment to Public Patient involvement (PPI) is based on ensuring our research results do enhance peoples' lives. So, following the needs assessment with all the stakeholders, it is vital to ensure that teens with JIA and their families retain a voice in the development of all materials and roll-out of the pilot RCT.

\section{STUDY MONITORING PROCEDURES}

In addition to the input from the YPAP, 'Lending an Ear' will have monthly meetings between all co-applicants and collaborators to ensure their expertise continues to inform challenge resolutions and progression.

\section{MEASURES}

\section{Feasibility outcomes}

Participant accrual and dropout rates will be centrally tracked online by postdoctoral researcher and RA.

\section{Fidelity}

Any issues or difficulties encountered during implementation of interventions, control strategy or outcome measures will be tracked.

\section{Acceptability and satisfaction with interventions}

- Post-treatment, adolescents with JIA and their parents in the TTC and iP2P-TTC intervention groups will rate acceptability of and satisfaction with the intervention online and through interviews (see below).

- Satisfaction with TTC and the integrated iP2P-TTC programme will also be captured using semistructured interviews at study completion with our to six adolescent-parent dyads (chosen via random numbers list). Broader assessment of engagement (eg, most helpful aspects, enjoyment, how tailoring was done) will be part of the semistructured interviews.

- All participants randomised to the integrated iP2PTTC will also be given a measure of mentor quality (Mentor Behaviour Scale) immediately following completion.

- Mentors will complete measures to assess their views on the iP2P training and invited to
- Individual semistructured telephone interviews conducted before they commence the mentoring programme (to gauge their expectations).

- Ecological Momentary Assessment methodology will be used throughout the mentoring programme. Mentors will be asked to complete a brief online open-ended questionnaire immediately after each mentee Skype call.

- Individual 'data-prompted' interviews conducted face-to-face once the mentoring programme is complete.

\section{Engagement with interventions}

- Google Analytics will track patterns of website programme usage by adolescents with JIA and parents (eg, which TTC modules have been accessed and in what order) in TTC group.

- For participants in the integrated iP2P-TTC intervention, the order and amount of TTC modules used, number and length of calls with mentor, and discussed topics will be tracked.

- Medical Issues, Exercise, Pain and Social Support Questionnaire (MEPS) ${ }^{17}$ questionnaire (see Effectiveness outcomes section) will provide information on improved knowledge.

\section{Tailoring}

To evaluate whether the iP2P component facilitates tailoring of the intervention to the needs of each teen, we will track for both intervention groups which modules they have visited and in which order. In addition, the semistructured interviews with mentors and mentees will ask more details on how exactly this tailoring took place.

\section{Effectiveness outcomes}

Adolescent will complete measures online evaluating

- Self-management (TRANSITION-Q) ${ }^{18}$ 3-point scale, 14 items.

- HRQL (PedsQL Arthritis Module) ${ }^{19}$ 5-point scale, five areas: Problem with Pain and Hurt (four items), Problems with Daily Activities (five items).

- Pain (PROMIS Paediatric Profile Pain Intensity and Interference scales) ${ }^{20} 5$-point scale (eight items).

- Revised Children's Anxiety and Depression Scale $^{21} 22-25$ items, with subscale scores for depression and anxiety as well as an overall internalising score.

- Disease knowledge (MEPS $)^{17}$ 10-point scale, four areas: Medical Issues (nine items), Exercise (four items), Pain (six items), Social Support (four items).

- Self-efficacy (Children's Arthritis Self-Efficacy) ${ }^{23}$ 5-point scale (11 items).

- Perceived social support (PROMIS Paediatric Profile Peer Relationship Scale) ${ }^{24}$ Short form 5-point scale (eight items).

- Health Services Use and Out-of-Pocket Expense Diary-Youth Version, ${ }^{25} 10$ different areas: Extracurricular activities (two items), Academic activities (six 
items), Loss of time (five items), Contact with medical doctor (five items), Allied health professionals and social service providers (2 Items), Emergency room visits (seven items), Hospital admissions (three items), Medication (two items), Medical devices (two items), Parent loss of time from work (pain or unpaid) (16 items). Measure was adapted from health economist's thesis project.

Parents will complete on-line measures assessing adolescents

- HRQL (PedsQL Arthritis Module). ${ }^{19}$

- Adherence (Adherence report questionnaire; PARQ),${ }^{26}$ one to seven measures, scale of 10.

- Medical issues (Medical Issues Questionnaire) ${ }^{17}$ (nine items).

- Self-efficacy (Parent Arthritis Self-Efficacy) ${ }^{23} 14$ measures, 0-7-point scale from very uncertain to certain.

Mentors will complete in relation to their own abilities

- Ability to Participate in Social Roles and ActivitiesPROMIS Short Form 8a-10-point scale (eight items): Exercise regularly scale (three items); Get information about disease (one item); Obtain help from community, family, friends (four items); Communicate with physician (three items); Manage disease in general (five items); Do chores (three items); Social/ recreational activities (two items); Manage symptoms (five items); Manage shortness of breath (one item); Control/manage depression (six items).

- Chronic Disease Self-Efficacy Scale, 5-point scale, 10 areas.

- PROMIS-29 Profile V.2.0, ${ }^{20}$ 5-point scale, eight areas: Physical function (four items), Anxiety (four items), Depression (four items), Fatigue (four items), Sleep disturbance (four items), Ability to participate in social roles and activities (four items), Pain interference (four items) and Pain intensity on a scale of 1-10 (one item). iPeer2Peer Mentor Training Evaluation 5-point scale, 10 items. ${ }^{11}$

- Post-intervention semistructured focus group to explore their perspectives on how well the programme worked for the mentees and themselves.

In addition to completing effectiveness outcome measures of TTC, mentees will also complete

- Mentor Behaviour Scale-5-point scale of four areas: Structure (eight items), Engagement (two items), Autonomy support (two items) and Competency support (three items). ${ }^{27}$

All measures have evidence of reliability and validity in samples of adolescents with JIA.

\section{BACKGROUND MEASURES}

For descriptive purposes and to obtain information on potential moderators of the strength of observed treatment effects, the following variables will be assessed at baseline:

- Adolescent and parent sociodemographic and JIArelated characteristics. Because this intervention is designed to be an adjunct to usual management approaches for JIA, participants will not be excluded if they are receiving common medical and physically based therapies. Information will be collected at each outcome measurement time point on whether participants in any group used or sought out any adjunct therapies (eg, medications, physical, psychological and complementary/alternative therapies), social support (eg, Facebook or Instant Messaging), diseasespecific information or attendance at a support group camp during the study period, to determine their extent of use.

- Access, use and comfort level with computers and the Internet (as expressed by themselves).

- Expectation about treatment effectiveness from adolescents and parents (using numerical rating scale-0 $0=$ "don't think it will help at all" to $10=$ "think it will help a lot").

\section{RETENTION AND ADHERENCE}

Adolescents will be encouraged to log onto the website once per week for 12 weeks and complete one module per week.

If a mentor/mentee dyad has not had a call within 2 weeks but has not indicated to the RA that they have terminated the programme, the RA will contact the participant to determine interest in continuing versus terminating their involvement or if they prefer to continue with just TTC programme. If they have decided to end the programme, they will be asked to fill out outcome measures. If scheduled calls are missed, participants will receive reminders by the mentor and/or the RA via email, text or phone. We will control for the number of Skype calls made in the analyses.

Characteristics of adherent versus non-adherent participants will be examined for systematic differences; when found, analyses will be conducted to determine effect on outcomes.

\section{Losses to follow-up}

Every effort will be made to retain participants and to obtain post-treatment measures on all who enrolled. We will ask for multiple phone numbers (home, mobile phone) and/or email addresses. To minimise losses to follow-up, the RA may make calls, texts and emails reminding participants of timing of various online assessments.

\section{DATA ANALYSIS}

All semistructured interviews will be audiotaped and transcribed verbatim to determine satisfaction with the Irish TTC or TTC+iP2P programme. ${ }^{6}$ The transcribed data will be managed using NVivo V.11 computer software program, which allows for online coding and annotation of text. We will use content analyses as outlined by Elo and Kyngäs. ${ }^{28}$ 
Quantitative data will be analysed using SPSS V.21. Descriptive statistics will be used to describe sample characteristics at baseline. Rates of accrual dropout, compliance and missing data with $95 \%$ CIs will be calculated. For continuous outcomes, the normality of the data will be assessed using histograms and normal probability plots. If assumptions are met, continuous outcomes will be analysed using linear mixed modelling to assess effect over time. If the data are not normally distributed, nonparametric equivalents will be used. For binary outcomes, a logistic regression analysis will be conducted.

To inform sample size calculations and data analysis for a larger trial, data will be analysed as in a larger study, and estimates of variance and correlation (ie, intracluster correlation within site) on physical (pain, fatigue) and emotional (anxiety, depression) symptoms, perceived social support, self-efficacy, adherence, knowledge and HRQL will all be estimated. Analysis will be conducted using an intent-to-treat approach. If assumptions for parametric statistics are met, linear mixed models will be used to test intervention effects on outcomes using an analysis of covariance approach with post-treatment measures compared between groups using baseline scores as covariates. To control for type 1 error rate, Holm's sequential correction will be applied. We will use the CONSORT $^{29}$ reporting guidelines to report this trial.

Cost-effectiveness and cost-utility analyses will be conducted using both a healthcare system and societal perspective. Cost-effectiveness and cost utility will be expressed as incremental cost-effectiveness ratios (ICERs), ${ }^{30}$ calculated by dividing the incremental costs between treatment arms by the incremental change in utility scores, measured as HRQL using the PedsQL. Multiple ICERS will be calculated comparing each of the three study groups in a pairwise fashion for both the costeffectiveness and cost-utility analyses. Extensive deterministic and probabilistic sensitivity analysis will be performed to evaluate the robustness of the results. A 95\% CI for incremental costs, incremental effects and the ICER will be calculated from study data using bootstrapping.

\section{DATA MONITORING AND MANAGEMENT}

This study will collect non-identifying, minimally invasive information, which is not expected to cause any level of distress to participants. All data will be collected electronically and stored securely at the CPR on passwordprotected databases that can only be accessed by the research team.

Should any participant-parent, adolescent or young adult-indicate at any point during the study that they no longer wish to participate, that decision will be respected. If they would like to have their data up to that point destroyed or not used in the final analyses, they can inform the researcher of their wishes and their data will be confidentially shredded.

\section{Ethical considerations}

If an adolescent is found to be at risk of becoming distressed or not taking their meds, appropriate methods will be taken to inform those in positions of authority. The guidelines of the Child Protection Policy outlined by the respective hospital where the adolescent is being treated will be followed to ensure that they are fully supported throughout the project in relation to any issues that may arise. If an adolescent is considered to be at risk during the course of the project, a senior clinical psychologist will be contacted by the researchers to provide appropriate guidance and consultation if necessary.

In accordance with the Ethical Guidance for research with children, all members of the research team will be Gardaí vetted and employment checks will be carried out.

All members of the research team will be trained and have access to relevant expertise in relation to child protection issues. All those researchers having face-toface contact with the adolescents will have taken part in Health Service Executive (HSE) Child First training.

Written consent and assent will be obtained from all participating adolescents, their parents and the young adult mentors. During the consent process, the study procedures will be described in detail to both parents and adolescents, giving time to read the information and opportunities to ask questions. All participants will be advised that they are able to stop their participation at any time.

\section{Dissemination}

We will use both integrative and end-of-project knowledge exchange approaches to disseminate the findings to the public, patients with JIA and their families, support organisations, researchers and clinicians.

Approach 1 will include

1. Involving key stakeholders in all stages of the research process from the outset. Key stakeholders include adolescents with JIA (represented by the YPAP, patient organisations and clinicians).

2. Presentation of research findings by the PI and coapplicants at National, European and International conferences, plus published in leading paediatric or rheumatology journals to target all practising HCPs.

3. Other strategies will include a 1-page report and YouTube video that will be

a. Distributed to rheumatology HCPs and patient groups across Ireland.

b. Included in media releases and posting/links on key websites (eg, http://www.juvenilearthritis.ie) and social media.

c. Sent to all participants at the end of the project to inform them of the findings.

d. Included in conference presentations, which will be co-presented by researchers and stakeholders (eg, adolescents with JIA, AI or iCAN representative) where possible.

Approach 2 will involve launching an interactive Internet conduit ('Irish Teens Taking Charge: Managing 
Arthritis Online' and peer mentoring) at the end of the project, if found to be effective, to share knowledge with users, predominately adolescents with JIA and their families, as well as other web audiences (eg, peers, teachers and health professionals).

This mode of communication can provide an effective tool to help in the collection, processing and targeted distribution of information about JIA research to benefit patients and their families, clinicians, researchers, administrators, healthcare policy-makers, school administrations and the public.

\section{Author affiliations}

${ }^{1}$ Centre for Pain Research, School of Psychology, National University of Ireland, Galway, Galway, Ireland

${ }^{2}$ Faculty of Nursing, University of Toronto, Toronto, Ontario, Canada

${ }^{3}$ Anesthesia and Pain Medicine, Hospital for Sick Children, Toronto, Ontario, Canada ${ }^{4}$ Medical Psychiatry Alliance, Hospital for Sick Children, Toronto, Ontario, Canada ${ }^{5}$ Division of Psychology, Faculty of Natural Sciences, University of Stirling, Stirling, UK

${ }^{6}$ School of Psychology, National University of Ireland, Galway, Ireland

Acknowledgements All our collaborators and funders. iCAN, Arthritis Ireland, all the families and HCPs who are working with us on this project.

Collaborators Team Pain, Centre for Pain Research, School of Psychology, NUI Galway: Laura 0'Connor, Brian Slattery, Michelle Hanlon, Hannah Durand, Stephanie Haugh.

Contributors SO'H: Applied for the ethics in both university and hospital, recruited both YPAPA and Mentors, trained both YPAP and mentors, devised recruitment posters, parents invite letter, consent and assent forms. Liaised with HCPs and families. Devised the layout of the measures and the survey for all participants. Tested same and made amendments. Wrote the paper and the protocol. Revised it critically for important intellectual content. Approved final version. Agrees to be accountable for all aspects of the work. JS: designed and rolled out Canadian TTC and iPeer2Peer. Oversaw all adaptions to the text and videos. Liaised with all HCPs and families. Helped to draft the paper and the protocol and revised it critically for important intellectual content. Gave final approval of the version published. Agrees to be accountable for all aspects of the work. SAK: designed and rolled out Canadian IP2P and oversaw adaptions to the training and planning of the mentoring aspect of the study. Helped design the measures for the whole study to ensure questionnaire not too onerous for participants. Revised the paper critically for important intellectual content. Gave final approval of the version published. Agrees to be accountable for all aspects of the work. LC: involved with the design of the work, helped revise and then deliver the adapted training for the mentors. Supported the drafting of the paper and also revised it critically for important intellectual content. Gave final approval of the version published. Agrees to be accountable for all aspects of the work. $\mathrm{CH}$ : involved in the design of the pilot RCT and selection of appropriate measures. Revised the paper critically for important intellectual content. Gave final approval of the version published. Agrees to be accountable for all aspects of the work. BEM: designed the whole study, oversaw all engagement with participants and stakeholders, advised on selection of appropriate measures. Has final say on all aspects of the study as PI. Revised the paper critically for important intellectual content. Gave final approval of the version published. Agrees to be accountable for all aspects of the work.

Funding This work is supported by Irish Health Research Board (HRB) and National Children's Hospital (NCHF), grant number-NCHF-2017-003.

Competing interests None declared.

Patient consent for publication Not required.

Ethics approval Research Ethics Committees-National University of Ireland, Galway and Our Lady's Children's Hospital (OLCH), Crumlin, and Temple Street Children's Hospital, Dublin.

Provenance and peer review Not commissioned; externally peer reviewed.

Open access This is an open access article distributed in accordance with the Creative Commons Attribution Non Commercial (CC BY-NC 4.0) license, which permits others to distribute, remix, adapt, build upon this work non-commercially, and license their derivative works on different terms, provided the original work is properly cited, appropriate credit is given, any changes made indicated, and the use is non-commercial. See: http://creativecommons.org/licenses/by-nc/4.0/.

\section{REFERENCES}

1 Arthritis Ireland (2017). Available: http://www.juvenilearthritis.ie

2 Sawyer MG, Whitham JN, Roberton DM, et al. The relationship between health-related quality of life, pain and coping strategies in juvenile idiopathic arthritis. Rheumatology 2004;43:325-30.

3 Stinson J, Ahola Kohut S, Forgeron P, et al. The iPeer2Peer program: a pilot randomized controlled trial in adolescents with juvenile idiopathic arthritis. Pediatr Rheumatol Online J 2016;14.

4 Simons LE, Logan DE, Chastain L, et al. Engagement in multidisciplinary interventions for pediatric chronic pain: parental expectations, barriers, and child outcomes. Clin J Pain 2010;26:291-9.

5 Stinson J, McGrath P, Hodnett E, et al. Usability testing of an online self-management program for adolescents with juvenile idiopathic arthritis. J Med Internet Res 2010;12:e30.

6 Stinson JN, McGrath PJ, Hodnett ED, et al. An Internet-based self-management program with telephone support for adolescents with arthritis: a pilot randomized controlled trial. J Rheumatol 2010;37:1944-52.

7 Dennis C-L. Peer support within a health care context: a concept analysis. Int J Nurs Stud 2003;40:321-32.

8 Kohut S, Stinson J. Systematic review of peer support interventions for adolescents with chronic illness: a narrative analysis. Int $J$ Child Adolescent Health 2014;7:183-97.

9 Sandhu S, Veinot P, Embuldeniya G, et al. Peer-to-peer mentoring for individuals with early inflammatory arthritis: feasibility pilot. BMJ Open 2013;3:e002267.

10 Zelikovsky N, Petrongolo J. Utilizing peer mentors for adolescents with chronic health conditions: potential benefits and complications. Pediatr Transplant 2013;30:n/a-591.

11 Ahola Kohut S, Stinson JN, Ruskin D, et al. iPeer2Peer program: a pilot feasibility study in adolescents with chronic pain. Pain 2016;157:1146-55.

12 Ford P. The APP route to patient engagement. HIMSS Europe 2017;5:16-17.

13 O'Sullivan G, O'Higgins S, Caes L, et al. Self-management needs of Irish adolescents with juvenile idiopathic arthritis (JIA): how can a Canadian web-based programme meet these needs? Pediatr Rheumatol Online J 2018;16:68.

14 Stinson J, O'Higgins S. Pushing the frontier of digital health to transform paediatric self-management (PowerPoint presentation to health care providers in Temple Street Children's Hospital, Dublin) 2019.

15 Hertzog MA. Considerations in determining sample size for pilot studies. Res Nurs Health 2008;31:180-91.

16 Arain M, Campbell MJ, Cooper CL, et al. What is a pilot or feasibility study? A review of current practice and editorial policy. BMC Med Res Methodol 2010;10:67.

17 André M, Hedengren E, Hagelberg S, et al. Perceived ability to manage juvenile chronic arthritis among adolescents and parents: development of a questionnaire to assess medical issues, exercise, pain, and social support. Arthritis Care Res 1999;12:229-37.

18 Klassen AF, Grant C, Barr R, et al. Development and validation of a generic scale for use in transition programmes to measure selfmanagement skills in adolescents with chronic health conditions: the TRANSITION-Q. Child Care Health Dev 2015;41:547-58.

19 Varni JW, Seid M, Smith Knight T, et al. The PedsQL ${ }^{T M}$ in pediatric rheumatology: reliability, validity, and responsiveness of the Pediatric Quality of Life Inventory ${ }^{\mathrm{TM}}$ Generic Core Scales and Rheumatology Module. Arthritis Rheum 2002;46:714-25.

20 Jacobson CJ, Kashikar-Zuck S, Farrell J, et al. Qualitative evaluation of pediatric pain behavior, quality, and intensity item candidates and the PROMIS pain domain framework in children with chronic pain. $J$ Pain 2015;16:1243-55.

21 Chorpita BF, Yim L, Moffitt C, et al. Assessment of symptoms of DSM-IV anxiety and depression in children: a revised child anxiety and depression scale. Behav Res Ther 2000;38:835-55.

22 Chorpita BF, Ebesutani C, Spence S. Revised children's anxiety and depression scale, user guide, 2015. Available: www.childfirst.ucla. edu [Accessed 22 Apr 2018].

23 Brady TJ. Measures of self-efficacy: arthritis self-efficacy scale (ases), arthritis self-efficacy Scale-8 item (ASES-8), children's arthritis self-efficacy scale (case), chronic disease self-efficacy scale (CDSES), parent's arthritis self-efficacy scale (PASE), an. Arthritis Care Res 2011;63:S473-85. 
24 Dewalt DA, Thissen D, Stucky BD, et al. PROMIS Pediatric Peer Relationships Scale: development of a peer relationships item bank as part of social health measurement. Health Psychol 2013;32:1093-103.

25 Moretti ME. A cost-effectiveness analysis of maternal genotyping to guide treatment for postpartum pain and avert infant adverse events. [PhD Dissertation]. Toronto, Canada: Institute of Health Policy Management and Evaluation, University of Toronto, 2014.

26 De Civita M, Dobkin PL, Ehrmann-Feldman D, et al. Development and preliminary reproducibility and validity of the Parent Adherence Report Questionnaire: a measure of adherence in juvenile idiopathic arthritis. J Clin Psychol Med Settings 2005;12:1-12.
27 Brodeur P, Larose S, Tarabulsy G, et al. Development and construct validation of the Mentor Behavior Scale. Mentor Tutor Partnership Learn 2015;23:54-75.

28 Elo S, Kyngäs H. The qualitative content analysis process. J Adv Nurs 2008;62:107-15.

29 Eldridge SM, Chan CL, Campbell MJ, et al. CONSORT 2010 statement: extension to randomised pilot and feasibility trials. BMJ 2016;355:i5239.

30 Bensink $\mathrm{M}$, Wootton $\mathrm{R}$, Irving $\mathrm{H}$, et al. Investigating the costeffectiveness of videotelephone based support for newly diagnosed paediatric oncology patients and their families: design of a randomised controlled trial. BMC Health Serv Res 2007;7:38. 